\title{
Communication skills assessment in the final postgraduate years to established practice: a systematic review
}

\author{
Amy E Gillis, ${ }^{1}$ Marie C Morris, ${ }^{2}$ Paul F Ridgway ${ }^{2,3}$
}

${ }^{1}$ Department of Surgery,

Tallaght Hospital, Tallaght, Dublin, Ireland

${ }^{2}$ Education Division, School of Medicine, University of Dublin, Trinity College, Dublin, Ireland ${ }^{3}$ Department of Surgery, University of Dublin, Trinity College, Tallaght Hospital Campus, Dublin, Ireland

\section{Correspondence to}

AE Gillis, Rm 1.36 Trinity Centre for Health Sciences, Tallaght Hospital, Dublin 24 Ireland;

agillis@tcd.ie

Received 15 May 2014

Accepted 12 November 2014

Published Online First

2 December 2014
CrossMark

To cite: Gillis $A E$, Morris MC, Ridgway PF. Postgrad Med J

2015;91:13-21.
ABSTRACT

Introduction Communication breakdown is a factor in the majority of all instances of medical error. Despite the importance, a relative paucity of time is invested in communication skills in postgraduate curricula. Our objective is to systematically review the literature to identify the current tools used to assess communication skills in postgraduate trainees in the latter 2 years of training and in established practice.

Methods Two reviewers independently reviewed the literature identifying communication skill assessment tools, for postgraduate trainees in the latter 2 years of training and in established practice following Preferred Reporting Items for Systematic Reviews and MetaAnalyses framework, and inclusion/exclusion criteria from January 1990 to 15 August 2014. Databases: PubMed/ CINAHL/ERIC/EMBASE/PsycInfo/Psyc Articles/Cochrane.

Results 222 articles were identified; after review, 34 articles fulfilled criteria for complete evaluation; the majority (26) had a high level of evidence scoring 3 or greater on the Best Evidence Medical Education guide. 22 articles used objective structured clinical examination/ standardised patient (SP)-based formats in an assessment or training capacity. Evaluation tools included authordeveloped questionnaires and validated tools. Nineteen articles demonstrated an educational initiative.

Conclusions The reviewed literature is heterogeneous for objectives and measurement techniques for communication. Observed interactions, with patients or SPs, is the current favoured method of evaluation using author-developed questionnaires. The role of selfevaluation of skill level is questioned. The need for a validated assessment tool for communication skills is highlighted.

\section{INTRODUCTION}

Communication breakdown is a factor in $>50 \%$ of all postoperative complications, $70 \%$ of all medication errors and $80 \%$ of delays in treatment that resulted in death or permanent loss of function for patients. $^{1}$ Poor communication can exist at any level of patient and healthcare worker interaction, and most errors occur in verbal communication. ${ }^{2}$ Awareness surrounding adverse events in healthcare is increasing, thought to be attributed to behavioural failures rather than failures in technical expertise. ${ }^{3}$ Litigation for medical error is increasing. Physicians who provide information, spend more time defining patient expectations and solicit patient opinion tend to have fewer malpractice claims and higher patient satisfaction. ${ }^{4}$ Communication skills and behavioural interactions have been recognised as important in other high- risk industries such as aviation, and protocols for training communication skills specifically with management teams during stressful events have been developed to address these behaviours. ${ }^{5}$

In medicine, communication skills are thought to be acquired primarily by observation and by modelling, generally without formal feedback or formal assessment. Integrating communication skills into programmes has been attempted in many forms. The use of single lectures, series lectures, workshops and simulations has been demonstrated. ${ }^{6-9}$ Communication is multidimensional, and assessment needs to include the indistinct aspects such as establishing the physician-patient relationship, empathy and non-verbal interaction. ${ }^{6}$

The Kalamazoo conference ${ }^{10}$ identified three methods for evaluating communication skills, namely checklists of observed behaviours, patient experience surveys and oral or written examinations. Checklists, objective structured clinical examination (OSCE) examinations with standardised patients (SPs) and observed interactions with real patients (whether recorded or observed in real time) are all methods that can be used to develop and test these skills within undergraduate and postgraduate training programmes.

It is recognised that medical education must function on a continuum from medical school onwards throughout a career. At different points, individuals progress through transitions (eg, from postgraduate training to subspecialty training) that require additional support and a rebalance in educational needs and responsibilities. ${ }^{11}$ Learning habits shift abruptly when exiting training programmes from an enforced curriculum to a self-directed one, for which trainees may be unprepared. ${ }^{11}$

With communication skills, these are emphasised in the undergraduate curriculum; however, attitudes towards communication skills development tend to diminish as medical students graduate to postgraduate training programmes. ${ }^{12}$ New postgraduate trainees are fresh from medical school with recent experience of a formalised curriculum, which includes communication skills training. As time progresses through postgraduate training, other necessary skills take priority over communication skills in the learning programme. A decline or erosion in communication skills has been noted over time in undergraduate students. ${ }^{13}$

In a review of risk communication in postgraduate programmes in the USA, only a median of $12 \mathrm{~h}$ was allotted to communication skills development. ${ }^{14}$ Similarly, a survey of accredited postgraduate oncology fellowship programs in the USA 
showed only $30 \%$ had some form of formal communication skills training. ${ }^{7}$ Though recognised as a core competency in medical training, ${ }^{15}$ barriers exist such as lack of faculty time and expertise. Imposing a mandatory core competency assessment is equally hampered by the lack of an accepted, validated method of assessment. ${ }^{7}$ With new graduates entering into the workforce and physicians from many countries migrating to new healthcare systems, it is necessary to ensure that communication skills are, at minimum, adequate to meet standards for the safe treatment of patients.

The objective of this study was to identify the methods used to evaluate communication skills in postgraduate training and in established practice. The focus is to examine the tools used in the latter 2 years of a primary postgraduate training programme, the point where graduates are potentially transitioning to practice and evaluation methods used for those in established practices. The methodological rigour of these studies will be assessed.

\section{METHODS}

\section{Search strategy}

A systematic review of the literature was conducted by two independent reviewers (AEG and MCM) following Preferred Reporting Items for Systematic Reviews and Meta-Analyses (PRISMA) guidelines. ${ }^{16} \mathrm{~A}$ comprehensive review of the following electronic databases was undertaken-PubMed/CINAHL/ ERIC/EMBASE/PsycInfo/Psyc Articles/Cochrane-using the search terms 'communication skills assessment', 'postgraduate', 'surgery', 'medicine' and all related derivations.

\section{Inclusion criteria}

All studies published in the English language, from January 1990 to 15 August 2014, were included. Studies required involvement of medical practitioners, physicians or surgeons within 2 years of completion of a primary postgraduate training programme or established practising consultants.

\section{Exclusion criteria}

Studies in which the participants were more than 2 years from completion of postgraduate training (early within the training scheme) were excluded. It was recognised that programmes within different training schemes have different end points (eg, family medicine programmes can be $2-3$ years in length, surgical programmes can extend from 5 to 8 years (or more) depending upon specialty and training scheme). Care was taken to include all articles that may fit inclusion criteria. Ambiguity on the postgraduate level was erred on the side of inclusion rather than exclusion. No exclusion criteria were placed on assessment methods; any form of communication and method of evaluation could be included.

\section{Criteria for assessing the quality of studies}

Each paper was assessed for inclusion/exclusion criteria and for quality of methodology by two independent reviewers (AEG and MCM) using the Best Evidence Medical Education (BEME) guide. ${ }^{17} \mathrm{~A}$ third reviewer (PFR) arbitrated for any disputes. Each article was assessed and graded for research design, clarity of aims and use of a control group. The use of objective evaluation methods and self-assessment tools was noted, specifically the rigour of testing preceding the use of these tools. For example, tools that have been validated and published with appropriate reliability evaluation were rated higher than authordeveloped questionnaires, without accompanying validation. Articles that relied solely on self-evaluation of participants' confidence in ability scored lower than studies that used objective evaluation with validated tools.

\section{RESULTS}

\section{Systematic review process}

A total of 2514 articles were identified through systematic review, 2292 articles were rejected on initial review (the majority did not involve medical personnel (1156) or communication skills assessment (728)), leaving 222 articles for abstract review. Following abstract review, 160 articles did not meet inclusion criteria, the majority did not include communication skills assessment (122), 9 were excluded for junior postgraduate year (PGY) status and 5 articles were excluded due to undergraduate status. Sixty-two articles remained for full review. Eleven further articles were identified from hand search of references, leaving 73 articles for full review. After full text review, 39 articles were excluded due to lack of communication skills assessment (21), non-medical or other medical personnel (eg, nursing) (3), early and unclear PGY status not meeting criteria (3) or the full paper was not yet published and only available in abstract form (8). Thirty-four articles were available meeting inclusion and exclusion criteria (figure 1).

\section{Study findings}

Populations

In total, 22 of the 34 articles were based in the USA, 4 in Canada, 3 in the UK, 3 in Europe (2 Belgium, 1 Netherlands), 1 in Israel and 1 in India. Eleven articles involved family/general practice/internal medicine, 8 articles involved surgical residents (orthopaedic, plastic surgery, general surgery), 5 involved oncology practitioners, 4 articles involved paediatricians, 3 involved sampling from multiple disciplines and 3 articles involved radiology residents, geriatric and palliative care fellows, and critical care fellows, respectively. At total of 1355 participants within these papers fit the inclusion criteria of within 2 years of completion of training programme or were in consultant practice (table 1).

\section{Communication assessment tools}

Twenty-two articles used standardised patients, ${ }^{6} \begin{array}{lllll}18 & 20 & 24-29 & 31\end{array}$ $33-35 \quad 37-3942-479$ involved OSCE or objective structured performance evaluation. ${ }^{6} 2024-2643-47$ The majority $(n=21)^{6}{ }^{18-22}$ $\begin{array}{llllll}25 & 27 & 29-38 & 42 & 44 & 46\end{array}$ involved self-assessment by questionnaire, usually an author-developed, content-specific questionnaire. Seven articles used self-assessment measures only, ${ }_{19} \quad 21 \quad 22 \quad 29$

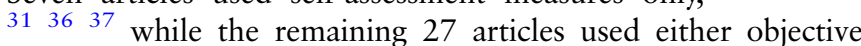
assessment by faculty/coworker and/or patient/standardised patient exclusively, or used in conjunction with self-assessment (table 2). Nineteen articles used an educational initiative to teach communication skills. ${ }^{6} 91921 \quad 27-31 \quad 33-3841424549$ The initiatives varied from lecture-based content, training programmes, to simulated patients' scenarios (table 3).

\section{Evaluation of methodological analysis}

The quality of the studies was variable, though the majority $(n=26)$ were grade 3 or above as scored by the BEME quality rating system. ${ }^{17}$ Rating of 3 or above ( 4 or 5 ) states the conclusions can probably be based on results or likely to be true based on the methodological rigour of the studies. The main weaknesses in the studies were the quality of the assessment tools and the presence of objective assessment. The majority of assessment tools (questionnaires) used were either developed by the author or the institution and were not previously validated within the literature or piloted prior to use. Those studies that 
Figure 1 Preferred Reporting Items for Systematic Reviews and Meta-Analyses (PRISMA) flow chart for article selection. PGY, postgraduate year.

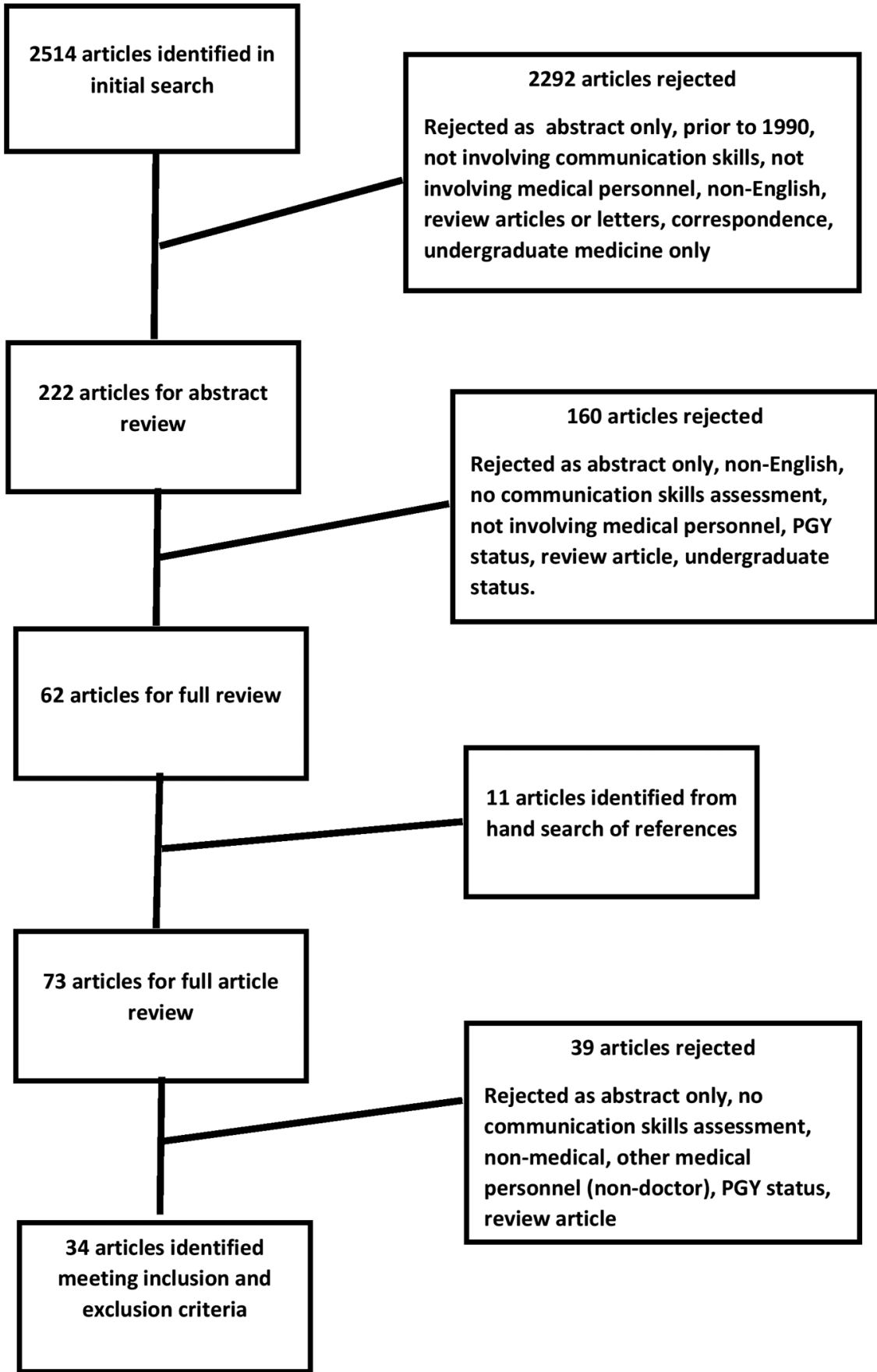

scored $<3$ generally relied upon participants' self-assessment of ability or confidence in ability. The reliability of self-assessment to correlate with actual ability is limited. As the majority of studies had a reasonable BEME rating, the findings can probably be based on the results found (table 2).

\section{DISCUSSION}

Communication within the healthcare system is of vital importance and remains one of the main factors of potential error that threatens patient safety. Though communication has been recognised by medical professional governance as a core competency for the professional, variability in how this skill set is taught and subsequently evaluated ${ }^{66} 67$ leads to heterogeneous tools of assessment highlighted in this review.

Observed interactions with either SPs or actual patients are the most often used techniques for communication skills assessment. The most robust studies have conducted objective evaluations using audio or video recording or real-time observations. These interactions have involved evaluation by expert facilitators or blinded coders using a previously validated checklist. Among these studies, there is no commonality with regards to checklist selection. Author-developed checklists were the most commonly used tools, and the majority are unvalidated. Within the studies identified, there is no standardisation among the groups of participants or scenarios, making comparison between the methodologies blunt. Self-evaluation is also common, which several authors have noted lacks correlation with objective assessment. ${ }^{30} 3246$ There is no standardised format available, nor was the exact criteria as to what constituted improved communication skills standardised. Variations from patient satisfaction, ${ }^{2} 4 \quad 9 \quad 11 \quad 19$ increased utterances of empathy, ${ }^{5} 19$ appropriate responses ${ }^{4} 88_{13-15} 192022$ and use of 
Table 1 Studies included in review

\begin{tabular}{|c|c|c|c|c|}
\hline \multirow[b]{2}{*}{ Study } & \multirow[b]{2}{*}{ Country } & \multicolumn{3}{|l|}{ Participants } \\
\hline & & Discipline & Total & PGY level \\
\hline Biernat et $a l^{18}$ & USA & Family medicine & $n=12$ & $n=9$ in year $2 / 3$ \\
\hline Krajewski et al ${ }^{19}$ & USA & General surgery & $n=43$ & no division by PGY level \\
\hline Lown et $a l^{20}$ & USA & Radiology & $\mathrm{n}=9$ & $\mathrm{n}=4 \mathrm{PGY} 4 / 5$ \\
\hline Singer and Carmel $^{21}$ & Israel & Family medicine & $n=15$ & Participants physicians from other countries \\
\hline Roter et al ${ }^{9}$ & USA & Internal medicine/family medicine & $n=15$ & $\begin{array}{l}\text { All established clinicians with 2-20 years' } \\
\text { experience }\end{array}$ \\
\hline Horowitz et $a l^{22}$ & USA & General surgery & $n=64$ & $\mathrm{n}=13$ PGY $4 / 5$ \\
\hline Grant et $a l^{23}$ & Canada & Paediatrics & $n=30$ & $n=12 P G Y 3 / 4$ \\
\hline Ponton-Carss et al $\left.\right|^{24}$ & Canada & General surgery & $n=14$ & $\mathrm{n}=2 \mathrm{PGY} 4$ \\
\hline Davis and Lee $2011^{25}$ & USA & Plastic surgery & $\mathrm{n}=6$ & $\mathrm{n}=4$ PGY $5 / 6$ \\
\hline Griesser et al ${ }^{26}$ & USA & Orthopaedic surgery & $n=22$ & No division by PGY level \\
\hline Wehbe-Janek et a/ ${ }^{6}$ & USA & General surgery & $n=14$ & $n=4$ PGY4 \\
\hline Razavi et al27 & Belgium & Oncology specialists & $n=62$ & $\mathrm{n}=62$ (established physicians) \\
\hline Levinson and Roter ${ }^{28}$ & USA & General internists/family practitioners & $n=50$ & $\mathrm{n}=50$ (established physicians) \\
\hline Baile et $a l^{29}$ & USA & Oncologists/oncology trainees & $n=12$ & $\mathrm{n}=12$ (established physicians and fellow level) \\
\hline Brown et a ${ }^{30}$ & USA & $\begin{array}{l}\text { Primary care physicians, surgeons, medical specialists, nursing } \\
\text { staff }\end{array}$ & $n=61$ & $\mathrm{n}=44$ \\
\hline Lenzi et $a^{\beta 1}$ & USA & $\begin{array}{l}\text { Fellows from oncology programmes (medical, surgical, } \\
\text { gynaecologic, etc) }\end{array}$ & $n=17$ & $\mathrm{n}=17$ \\
\hline Didwania et $a l^{32}$ & USA & Internal medicine residents & $\mathrm{n}=85$ & $\mathrm{n}=38$ in $\mathrm{PGY} 2$ \\
\hline Downar et $a l^{33}$ & Canada & Senior-level critical care trainees & $n=51$ & $n=51$ \\
\hline Elwyn et al ${ }^{34}$ & UK & General practitioners & $n=21$ & $\mathrm{n}=21$ \\
\hline Fallowfield et $a^{35}$ & UK & Oncologists & $\mathrm{n}=160$ & $n=160$ \\
\hline Farber et $a l^{\beta 6}$ & USA & Categorical medicine and Emergency medicine residents & $n=15$ & $n=8(n=7$ PGY2; $n=1$ PGY3) \\
\hline Geeta et $a l^{37}$ & India & Paediatric residents & $n=20$ & $\mathrm{n}=11$ (no PGY division; $\mathrm{n}=9$ PGY 1) \\
\hline Gelfman et $a l^{38}$ & USA & Geriatrics and palliative care fellows & $\mathrm{n}=9$ & $\mathrm{n}=9$ \\
\hline Griffith et $a^{\beta 9}$ & USA & Internal medicine residents & $n=59$ & $\mathrm{n}=47$ (no PGY division; $\mathrm{n}=12$ PGY 1) \\
\hline Howells et $a{ }^{40}$ & UK & Paediatricians & $n=19$ & $\begin{array}{l}\mathrm{n}=19 \text { (established physicians or advanced specialist } \\
\text { registrar status) }\end{array}$ \\
\hline Hulsman et $a l^{41}$ & Netherlands & Internists, surgeons, radiotherapists, etc & $n=21$ & $\mathrm{n}=21$ (established practitioners) \\
\hline Lagan et $a l^{42}$ & USA & Orthopaedic residents, nurse participants & $n=27$ & $\mathrm{~N}=18$ (no PGY division; mean years PGY 3) \\
\hline Parish et $a l^{43}$ & USA & Internal medicine/family medicine residents & $\mathrm{n}=265$ & $\mathrm{n}=265$ (all PGY3) \\
\hline Parish et al ${ }^{44}$ & USA & Internal medicine/family medicine residents & $n=131$ & $\mathrm{n}=131$ (all PGY3) \\
\hline Back et $a /^{45}$ & USA & Oncology fellows & $n=106$ & $n=106$ \\
\hline Falcone et $a l^{46}$ & USA & General surgery residents & $n=54$ & $\mathrm{n}=27$ (senior level=>PGY3) \\
\hline Jefferies et $a l^{47}$ & Canada & Neonatal perinatal fellows & $n=24$ & $\mathrm{n}=24$ \\
\hline Johnson et $a l^{48}$ & USA & Primary care physicians & $n=61$ & $\mathrm{n}=61$ (established practitioners) \\
\hline Lienard et $a^{/ 49}$ & Belgium & $\begin{array}{l}\text { Residents from varying training programmes: oncology, surgery, } \\
\text { gastroenterology, etc }\end{array}$ & $n=88$ & $\begin{array}{l}\mathrm{n}=10 \text { (PGY4/5; lack of specification of training } \\
\text { programme) }\end{array}$ \\
\hline
\end{tabular}

PGY, postgraduate year (number of years following completion of medical school).

increased non-verbal gestures ${ }^{9}{ }^{19}$ were all included as endpoints constituting improved communication. This review highlights a gap within the literature, namely that a single or series of objective assessment tools that have been widely used and evaluated does not exist.

These same issues are carried to those tools used in the development and evaluation of educational programmes. Though this review was not designed to assess the educational programmes, a number of tools were identified that used communication skill assessment techniques. Educational initiatives to teach communication skills were noted in 19 of the gathered articles. These initiatives were varied, involving lecture-based teaching, group workshops and/or role-play/scenario teaching. The majority of educational programmes used role-play in small groups with standardised or actual patients. The length of the programmes was unstandardised, from several hours to several days. Three computerised programs for analysing utterances within audio data were noted as an objective measure of communication skill and have been validated and used in many studies. ${ }^{28} 354849$ Self-assessment was again noted as a common measurement technique of evaluating the effectiveness of a programme by demonstrating a change in communication skills. Self-evaluation is inherently inaccurate. Pre-existing confidence in one's ability is not necessarily an accurate measure of competence. It has been pointed out: "...the worst self-assessment accuracy appears to be among physicians who are, at the same time, the least skilled and the most confident." ${ }^{68}$ From the 19 studies noted, there was no consensus on whether teaching communication skills actually improves communication, whether evaluation was purely objective or included self-evaluation. Again, the studies used different measurements of what constituted improved communication, which made direct comparison difficult. Those studies that reported some objective improvement in skill

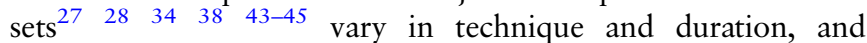


Table 2 Methods of assessment of communication skills

\begin{tabular}{|c|c|c|c|c|}
\hline Study & Use of OSCE/SP & Self-assessment & Objective measures & $\begin{array}{l}\text { BEME } \\
\text { score }\end{array}$ \\
\hline Biernat et $a l^{18}$ & SP & Yes-dementia interview checklist & $\begin{array}{l}\text { Yes-two independent experts using dementia } \\
\text { interview checklist form }\end{array}$ & 4 \\
\hline $\begin{array}{l}\text { Krajewski } \\
\text { et } a l^{19}\end{array}$ & No & Yes-author-developed questionnaire (pre/post) & No & 2 \\
\hline Lown et $\left.a\right|^{20}$ & OSCE with SP & Yes-author-developed questionnaire & Evaluation by expert with RCSAT & $3-4$ \\
\hline $\begin{array}{l}\text { Singer and } \\
\text { Carmel }^{21}\end{array}$ & No & Yes-author-developed questionnaire & No & 2 \\
\hline Roter $^{9}$ & No & No & $\begin{array}{l}\text { Yes-patient satisfaction assessed with questionnaire, } \\
\text { audiotapes analysed by independent experts }\end{array}$ & 4 \\
\hline Horwitz et $a l^{22}$ & No & Yes-SSI ${ }^{22}$ & No & 3 \\
\hline Grant et $a l^{23}$ & No & No & $\begin{array}{l}\text { Yes-Videotaped analysis of scenario using } \\
\text { leadership tool (three raters) and fourth blinded rater } \\
\text { (*communication a single component of entire } \\
\text { evaluation) }\end{array}$ & 5 \\
\hline $\begin{array}{l}\text { Ponton-Carss } \\
\text { et } a l^{24}\end{array}$ & OSCE with SP & No & $\begin{array}{l}\text { Yes-evaluated by consultants, communication } \\
\text { experts, co workers, patients with validated } \\
\text { checklist }^{10}\end{array}$ & 4 \\
\hline $\begin{array}{l}\text { Davis and } \\
\text { Lee }^{25}\end{array}$ & OSCE with SP & $\begin{array}{l}\text { Yes-author-developed written assessment } \\
\text { post-OSCE }\end{array}$ & $\begin{array}{l}\text { Yes-assessment of communication by SP using } \\
\text { author-developed scale }\end{array}$ & $3-4$ \\
\hline Griesser et $a l^{26}$ & OSCE with SP & No & $\begin{array}{l}\text { Yes-assessment of communication by SP using } \\
\text { validated score card }\end{array}$ & $3-4$ \\
\hline $\begin{array}{l}\text { Wehbe-Janek } \\
\text { et } a l^{6}\end{array}$ & OSCE with SP & $\begin{array}{l}\text { Yes-preauthor/postauthor-developed } \\
\text { questionnaire }\end{array}$ & $\begin{array}{l}\text { Yes-objective evaluation by coworkers, faculty, } \\
\text { peers, SP using author-developed questionnaires }\end{array}$ & 3 \\
\hline Razavi et $a l^{27}$ & $\begin{array}{l}\text { Training scenario with trained } \\
\text { SP and actual patient interviews }\end{array}$ & Yes-perception of interview questionnaire ${ }^{51}$ & $\begin{array}{l}\text { Yes-transcribed simulated and actual patient } \\
\text { interviews, evaluated by } 14 \text { trained psychologists with } \\
\text { validated tool }\end{array}$ & 4 \\
\hline $\begin{array}{l}\text { Levinson and } \\
\text { Roter }^{28}\end{array}$ & $\begin{array}{l}\text { Long course involves interview } \\
\text { of patients (SP) }\end{array}$ & No & $\begin{array}{l}\text { Yes-audiotaped patient sessions coded by blinded } \\
\text { judges using RIAS }{ }^{53}\end{array}$ & 4 \\
\hline Baile et $a l^{29}$ & Training involved SP interviews & $\begin{array}{l}\text { Yes-three questionnaires self-appraisal of abilities } \\
1 \text {-author developed } \\
\text { 2-previously validated: Outcome expectancies } \\
\text { measure, confidence in interviewing skills } \\
\text { questionnaire }\end{array}$ & No & 2 \\
\hline Brown et $a l^{30}$ & $\begin{array}{l}\text { No-dialogue with other } \\
\text { participants in training course }\end{array}$ & $\begin{array}{l}\text { Yes-self-assessment questionnaire } \\
\text { preintervention/postintervention }\end{array}$ & $\begin{array}{l}\text { Yes-patients rating of clinicians' communication } \\
\text { skills using the Art of Medicine Survey }{ }^{55}\end{array}$ & 4 \\
\hline Lenzi et $a l^{31}$ & Yes-sP & $\begin{array}{l}\text { Yes-self-assessment of confidence in performing } \\
\text { different communication tasks; evaluation of the } \\
\text { course by participants }\end{array}$ & No & $2-3$ \\
\hline $\begin{array}{l}\text { Didwania } \\
\text { et } a l^{32}\end{array}$ & No & $\begin{array}{l}\text { Yes-self-assessment of confidence- } \\
\text { author-developed questionnaire }\end{array}$ & $\begin{array}{l}\text { Yes-audiorecorded interactions and assessed by } \\
\text { primary faculty rater ( } 40 \% \text { checked by second blinded } \\
\text { rater) using published checklist: Handoff CEX }{ }^{56} 57\end{array}$ & $3-4$ \\
\hline Downar et $a l^{33}$ & $\begin{array}{l}\text { Yes—-standardised family } \\
\text { members }\end{array}$ & $\begin{array}{l}\text { Yes-tests on understanding ethical/legal } \\
\text { principles and participant comfort with } \\
\text { communication challenges pre/post; self-evaluation } \\
\text { of performance }\end{array}$ & $\begin{array}{l}\text { Yes-evaluation by standardised family members and } \\
\text { facilitators with author-developed evaluation tool } \\
\text { (reliability calculated Cronbach's } \alpha 0.6-0.9 \text { ) }\end{array}$ & 3 \\
\hline Elwyn et $a l^{34}$ & $\begin{array}{l}\text { Yes-during training sessions } \\
\text { and actual patient interactions }\end{array}$ & $\begin{array}{l}\text { Yes-author-developed questionnaire on clinician } \\
\text { perceptions of level of clinician-patient interaction }\end{array}$ & $\begin{array}{l}\text { Yes-audiotaped consultations with patients and } \\
\text { scored by independent researchers using the OPTION } \\
\text { scale }^{58}\end{array}$ & 4 \\
\hline $\begin{array}{l}\text { Fallowfield } \\
\text { et } a l^{35}\end{array}$ & $\begin{array}{l}\text { Yes-simulated patients during } \\
\text { training sessions, and actual } \\
\text { patient interaction }\end{array}$ & $\begin{array}{l}\text { Yes-reported in a separate article not included in } \\
\text { this study }\end{array}$ & $\begin{array}{l}\text { Yes-videotaped interaction, analysis using } \text { MIPS }^{59} \\
\text { by two independent raters }\end{array}$ & 4 \\
\hline Farber et $a l^{36}$ & No-_actual patient interactions & $\begin{array}{l}\text { Yes-author-developed prequestionnaire/ } \\
\text { postquestionnaire }\end{array}$ & No & 1 \\
\hline Geeta et $a l^{37}$ & Yes-SP 'trained volunteers' & $\begin{array}{l}\text { Yes-author-developed prequestionnaire/ } \\
\text { postquestionnaire }\end{array}$ & No & 1 \\
\hline Gelfman et $\left.a\right|^{38}$ & $\begin{array}{l}\text { Yes-SP involved in Geritalk }{ }^{60} \\
\text { training course; actual family } \\
\text { members involved in evaluation }\end{array}$ & Yes-author-developed pre/post questionnaire & $\begin{array}{l}\text { Yes-author-developed assessment tool (FaMCAT) } \\
\text { pre/post }\end{array}$ & 2 \\
\hline Griffith et $a l^{39}$ & Yes-SP & No & $\begin{array}{l}\text { Yes-author-developed case-specific checklists, } \\
\text { non-verbal skill global rating scale and checklist on } \\
\text { patient satisfaction, all completed by the SP for three } \\
\text { standardised patient encounters }\end{array}$ & 3 \\
\hline Howells et $a l^{40}$ & No-actual patient encounters & No & $\begin{array}{l}\text { Yes-development of a tool- PCAT- to measure } \\
\text { doctor-patient, doctor-child communication; video } \\
\text { recordings of interactions, rating by independent } \\
\text { raters }\end{array}$ & 5 \\
\hline
\end{tabular}


Table 2 Continued

\begin{tabular}{|c|c|c|c|c|}
\hline Study & Use of OSCE/SP & Self-assessment & Objective measures & $\begin{array}{l}\text { BEME } \\
\text { score }\end{array}$ \\
\hline $\begin{array}{l}\text { Hulsman } \\
\text { et } a l^{41}\end{array}$ & No & No & $\begin{array}{l}\text { Yes-independent assessment of video recordings } \\
\text { with } \mathrm{CRS}^{61} \text { by blinded raters; patient satisfaction } \\
\text { measured with } \text { MISS }^{62}\end{array}$ & 4 \\
\hline Lagan et $a l^{42}$ & $\begin{array}{l}\text { Yes_-SP during training } \\
\text { sessions }\end{array}$ & $\begin{array}{l}\text { Yes-author-developed prequestionnaire/ } \\
\text { postquestionnaire }\end{array}$ & $\begin{array}{l}\text { Yes-SP evaluation of participants during the three } \\
\text { encounters with University of Illinois Chicago-CIS } \\
\text { scale }^{63}\end{array}$ & 3 \\
\hline Parish et $a l^{43}$ & $\begin{array}{l}\text { Yes-OSCE with SP (five } \\
\text { stations) }\end{array}$ & $\begin{array}{l}\text { Resident assessment of the OSCE learning } \\
\text { experience }\end{array}$ & $\begin{array}{l}\text { Yes-faculty evaluation with author-developed global } \\
\text { rater tool; SP provided written comments of resident } \\
\text { performance }\end{array}$ & 3 \\
\hline Parish et $a l^{44}$ & $\begin{array}{l}\text { Yes-OSCE with SP (five } \\
\text { stations) }\end{array}$ & $\begin{array}{l}\text { Yes-assessment of competence in area of } \\
\text { substance abuse management (author-developed } \\
\text { questionnaire) }\end{array}$ & $\begin{array}{l}\text { Yes-faculty evaluation with author-developed global } \\
\text { rater tool; SP gave global satisfaction rating. }\end{array}$ & 3 \\
\hline Back et $a l^{45}$ & Yes-SP & No & $\begin{array}{l}\text { Yes-audio recordings of four SP encounters (two } \\
\text { pre/two post) using author-developed coding scheme } \\
\text { by trained blinded coders. ( } 10 \% \text { coded twice for } \\
\text { reliability) }\end{array}$ & 4 \\
\hline Falcone et $a l^{46}$ & $\begin{array}{l}\text { Yes-OSCE with SP (two } \\
\text { stations) }\end{array}$ & $\begin{array}{l}\text { Yes-author-developed questionnaire } \\
\text { self-assessment of confidence/experience }\end{array}$ & $\begin{array}{l}\text { Yes-SP evaluation using internally validated } \\
\text { checklist }^{64}\end{array}$ & 2 \\
\hline Jefferies et $a l^{47}$ & $\begin{array}{l}\text { Yes-OSCE with SP (10 stations } \\
\text {-for full CanMEDS } \\
\text { competencies) }\end{array}$ & No & $\begin{array}{l}\text { Yes_faculty examiners used author-developed } \\
\text { checklist (global rater); SP completed } \\
\text { author-developed global rater }\end{array}$ & 3 \\
\hline Johnson et $a l^{48}$ & No-actual patient interactions & No & $\begin{array}{l}\text { Yes-audio recordings of communications analysis } \\
\text { with RIAS coded tape }{ }^{53}\end{array}$ & 4 \\
\hline Lienard et $a l^{49}$ & $\begin{array}{l}\text { No-involving actual patient } \\
\text { encounters }\end{array}$ & No & $\begin{array}{l}\text { Yes-audio recordings of communication analysed } \\
\text { with LaComm software; patient satisfaction with } \\
\text { resident communication, author-developed global } \\
\text { rater }\end{array}$ & 4 \\
\hline
\end{tabular}

evaluation, leaving an agreement on the ideal scheme for communication skills development as yet unidentified.

Two minor issues were noted that could be of interest in future studies of communication skills. The study of Horwitz et $a l^{22}$ highlighted a potential use for the Social Skills Inventory (SSI). SSI is a previously validated and refined tool for generating a profile of social skills and has been extensively used within a variety of literature bases. Horwitz et $a l^{22}$ found that an imbalance in composite social skill profile could be elicited, indicating a potential communication skills deficit. This raises the question of whether this tool could be used to identify trainees and physicians with an imbalance of social skill profile. It may be a useful tool in establishing correlative communication deficiencies that can be targeted for improvement.

The second issue is a question of acquisition or deterioration of communication skill over years of training. Conflicting conclusions were made between several papers. ${ }^{21} 242647$ Two articles $^{24} 25$ noted a trend of an inverse association of worsening communication skill with increasing PGY level and suggest that this implies communication skills are inherent and not taught. Two further articles ${ }^{26} 47$ show a positive association with advancing PGY level and increased communication skills. The participant groups examined by four other groups 9223246 noted that no significant difference was found between communication skill and PGY level or years of experience. The variations in assessment and the stipulations of what constitutes an improvement in communication skill would likely account for the differences, and conclusions on whether learning or inherent skill is the primary driver of communication fluency cannot be made. However, the argument that providing insight into a deficit (of interpersonal communication skill) and providing a structured correctional programme of exposure and practice could seemingly provide for discrepancies in natural skill.

\section{Limitations}

This review is limited by the small number of studies meeting inclusion criteria and by the heterogeneity of the published papers. Each of the reviewed studies is affected by a number of limitations and biases. The majority of these studies recruit volunteers creating a bias towards those interested in improving or assessing their own communication skills. Those participating show insight and have an interest in communication skills that is often not present in those that have poor communication skills. The 'Hawthorne effect', ${ }^{69}$ where a participant's behaviour can improve or change in response to being observed, is a prevalent confounder in many of these studies with external observation. The halo effect or examiner bias could be existent as a number of evaluations are performed within residency programmes and examiners are likely to know or to have had some prior knowledge of the participants.

Also, search terms focused on communication skills assessment specifically; however, other associated concepts such as empathy, teamwork, professionalism and leadership were not included, but may have communication as a unifying theme that would not be represented within the search provided here.

This review highlights the heterogeneity within the literature surrounding communication skills and the assessment of these skills at the postgraduate level of training and beyond. It has highlighted the more common tools and methods of evaluation used in the latter stages of training and in practice. Many tools exist, though no one method or methods have become the 
Table 3 Educational initiatives

\begin{tabular}{|c|c|}
\hline Study & Description \\
\hline Krajewski et al ${ }^{19}$ & 2 didactic lectures-total $3 \mathrm{~h}$ \\
\hline Singer and Carmel $^{21}$ & 8-month training programme-end-of-life care, palliative training \\
\hline Roter et $a l^{9}$ & $4 \mathrm{~h} / 8 \mathrm{~h}$ role-play session of communication scenarios \\
\hline Wehbe-Janek et a/ ${ }^{6}$ & Communication scenarios with SP \\
\hline Razavi et $a l^{27}$ & $\begin{array}{l}\text { Basic training programme }(2 \times 8 \mathrm{~h} \text { day sessions and } 1 \times 3 \mathrm{~h} \text { session-consolidation workshop }(2 \mathrm{~h} \text { didactic lecture, } 17 \mathrm{~h} \text { small group playing sessions, } \\
2 \text { handbooks) - simulated interviews in small group sessions }\end{array}$ \\
\hline Levinson and Roter ${ }^{28}$ & $\begin{array}{l}\text { Short course: expert-led programme, didactic presentation and case-based discussions. Skills taught with patient-centred style of interviewing } \\
\text { Long course: small groups with facilitator, learner-centred learning, participants interview patients, with immediate feedback }\end{array}$ \\
\hline Baile et $a l^{29}$ & $\begin{array}{l}\text { Workshop: } 2.5 \text { days-didactic lecture, small groups with patient interviews and role-play, personal awareness exercise, group discussion/ } \\
\text { presentations }\end{array}$ \\
\hline Brown et $a l^{30}$ & $\begin{array}{l}2 \text { workshops: } 4 \mathrm{~h} \text { in duration, with } 2 \mathrm{~h} \text { of homework between workshops. Skills of listening, building relationships and negotiation, managing } \\
\text { disagreements }\end{array}$ \\
\hline Lenzi et $a l^{31}$ & $\begin{array}{l}3.5 \text { days-included lectures, large group exercises, simulated patient encounters and role-playing with experienced facilitator; literature provided at } \\
\text { commencement of the course }\end{array}$ \\
\hline Downar et $a l^{33}$ & Workshop: half day, 60 min didactic session and printed material; $4 \times 45$ min simulated family meetings with immediate feedback \\
\hline Elwyn et $a l^{34}$ & $\begin{array}{l}\text { Separate workshops on SDM and risk communication. SDM: simulated patient consultations with immediate feedback, presentations and } \\
\text { discussions. Risk communication: visual displays of risk estimates, practised communication with standardised patients with immediate feedback }\end{array}$ \\
\hline Fallowfield et $a l^{35}$ & $\begin{array}{l}\text { 3-day residential course-small groups led by facilitator with simulated patients with immediate feedback. Two separate interactions filmed for } \\
\text { analysis }\end{array}$ \\
\hline Farber et $a l^{36}$ & Interaction with a patient giving bad news, 15 min role-play with supervision and feedback from the patient to the resident with discussion. \\
\hline Geeta et $a l^{37}$ & Workshop $8 \mathrm{~h}$ duration involving lectures, group discussion and role-play \\
\hline Gelfman et $a l^{38}$ & Geritalk communication skills course ${ }^{60}$ didactic presentations, small group communication skills practice with simulated patients/family members \\
\hline Hulsman et al ${ }^{41}$ & 'Interact cancer' ${ }^{\prime 65}$ four computer-assisted modules dedicated to communication skills development; each module takes $1 \mathrm{~h}$ to complete \\
\hline Lagan et $a l^{42}$ & Clinician-patient workshop: $4 \mathrm{~h}$ - didactic discussion, small group simulated scenarios with SPs \\
\hline Back et al ${ }^{45}$ & 'Oncotalk'-4-day workshop emphasising small group role-play with facilitator and SP at different points along the cancer trajectory \\
\hline Lienard et $a l^{49}$ & BIC-CST-40 h (30 h training-role-play, $10 \mathrm{~h}$ stress management) \\
\hline
\end{tabular}

BIC-CST, Belgian Interuniversity Curriculum-Communication Skills Training; SDM, shared decision making; SP, standardised patient.

standardised means to assess this skill set. Much work needs to be done. A standardised format for evaluation of communication skills needs to be developed. The ideal educational programme, duration, method and conduct needs to be defined. This review has also identified the need for a defined, agreed standard of what constitutes acceptable communication skills.

\section{Main messages}

No definitive, validated tool to evaluate communication skills exists at the postgraduate level.

- Development of a validated tool is needed to progress standardised communication skills assessment at this level.

- The benefit of educational initiatives for improving communication skills cannot be demonstrated from the available literature.

- A defined standard of what constitutes acceptable communication skills needs to be defined.

\section{Current research questions}

What is the ideal educational programme to teach communication skills?

- Does the use of a social skills tool assist in identifying people in need of further communication skill training?

- Do communication skills ameliorate or deteriorate with advancing postgraduate levels?

\section{Key references}

- Roter D, Rosenbaum J, de Negri B, et al. The effects of a continuing medical education program in interpersonal communication skills on doctor practice and patient satisfaction in Trinidad and Tobago. Med Educ 1998;32:181-9.

- Ponton-Carss A, Hutchison C, Violato C. Assessment of communication, professionalism and surgical skills in an objective structured performance-related examination (OSPRE): A psychometric study. Am J Surg 2011;202:433-40.

- Razavi D, Merckaert I, Marchal S, et al. How to optimize physicians' communication skills in cancer care: results of a randomized study assessing the usefulness of post training consolidation workshops. J Clin Oncol 2003;21:3141-9.

- Levinson W, Roter D. The effects of two continuing medical education programs on communication skills of practicing primary care physicians. J Gen Intern Med 1993;8:318-24.

The quality of the studies and the innovative and novel techniques demonstrated by the studies included in this review indicate that much promise exists in finding an accurate means of furthering this necessary competency in medical education.

Contributors MCM and AEG conducted the development and implementation of the project design, acquired and interpreted the data. PFR assisted on design of the study and on analysis and interpretation of the data. All authors contributed to either writing or re-drafting this paper. All authors have reviewed this paper in its final version prior to submission with approval.

Competing interests None.

Provenance and peer review Not commissioned; externally peer reviewed. 


\section{REFERENCES}

1 The Joint Commission. Sentinel Event Statistics (Root Causes Event). 2004-2012 (cited 4 July 2013). http://www.jointcommission.org

2 Greenberg CC, Regenbogen SE, Studdert DM, et al. Patterns of communication breakdowns resulting in injury to surgical patients. J Am Coll Surg 2007;204:533-40.

3 Yule S, Paterson-Brown S. Surgeons' non-technical skills. Surg Clin N Am 2012:92:37-50.

4 Levinson W, Roter DL, Mullooly JP, et al. Physician-patient communication: the relationship with malpractice claims among primary care physicians and surgeons. JAMA 1997;277:553-9.

5 O'Connor P, Flin R, Fletcher G. Methods used to evaluate the effectiveness of CRM training: a literature review. J Hum Factors Aerospace Saf 2002:2:217-34.

6 Wehbe-Janek H, Song J, Shabahang M. An evaluation of the usefulness of the standardized patient methodology in the assessment of surgery residents' communication skills. J Surg Educ 2011;68:172-7.

7 Hoffman M, Ferri J, Sison C, et al. Teaching communication skills: an AACE survey of oncology training programs. J Cancer Educ 2004;19:220-4.

8 Madan AK, Caruso BA, Lopes JE, et al. Comparison of simulated patient and didactic methods of teaching HIV risk assessment to medical residents. Am J Prev Med 1998;15:114-19.

9 Roter D, Rosenbaum J, de Negri B, et al. The effects of a continuing medical education program in interpersonal communication skills on doctor practice and patient satisfaction in Trinidad and Tobago. Med Educ 1998:32:181-9.

10 Duffy FD, Gordon GH, Whelan G, et al. Assessing competence in communication and interpersonal skills: the Kalamazoo II report. Acad Med 2004;79:495-507.

11 Lockyer J, Silver I, Oswald A, et al. Competence by design: reshaping Canadian medical education -the continuum of medical education. $R$ Coll Phys Surg Can 2014;130-141 [cited 10 September 2014) http://www.royalcollege.ca/portal/page/ portal/rc/advocacy/educational initiatives/competence by design

12 Morris $\mathrm{M}$, Donohoe $\mathrm{G}$, Hennessy $\mathrm{M}$. The fall and rise of medical students' attitudes to communication skills learning in Ireland: a longitudinal approach. $\mathrm{Br} J$ Educ SoC Behav Sci 2013:3:467-77.

13 Kramer AWM, Dusman H, Tan LHC, et al. Acquisition of communication skills in post graduate training for general practice. Med Educ 2004;38:158-67.

14 Lefevre FV, Waters T, Budetti PP. A survey of physician training programs in risk management and communication skills for malpractice prevention. J Law Med Ethics 2000;28:258-66

15 Frank JR, ed. The CanMEDS 2005 physician competency framework. Better Standards. Better Physicians. Better care. Ottawa: The Royal College of Physicians and Surgeons of Canada, 2005 (cited 4 July 2013). http://www.royalcollege.ca/portal/page/portal/rc/ common/documents/canmeds/resources/publications/framework full_e.pdf

16 Moher D, Liberati A, Tetzlaff J, et al. Preferred reporting items for systematic reviews and meta-analyses: the PRISMA statement. BMJ 2009:339:332-6.

17 Harden RM, Grant J, Buckley G, et al. BEME guide 1: best evidence medical education. Med Teach 1999:21:553-62.

18 Biernat K, Simpson D, Duthie E Jr, et al. Primary care residents self-assessment skills in dementia. Adv Health Sci Educ 2003;8:105-10.

19 Krajewski A, Rader C, Voytovich A, et al. Improving Surgical Residents' Performance on written assessments of cultural competency. J Surg Educ 2008;65:263-9.

20 Lown BA, Sasson JP, Hinrichs P. Patients as partners in radiology education: an innovative approach to teaching and assessing patient-centred communication. Acad Radiol 2008;15:425-32.

21 Singer $Y$, Carmel $S$. Teaching end-of-life care to family medicine residents- what do they learn? Med Teach 2009;31:e47-50.

22 Horwitz IB, Horwitz SK, Brandt ML, et al. Assessment of communication skills of surgical residents using the Social Skills Inventory. Am J Surg 2007;194:401-5.

23 Grant EC, Grant VJ, Bhanji F, et al. The development and assessment of an evaluation tool for pediatric resident competence in leading simulated pediatric resuscitations. Resuscitation 2012;83:887-93.

24 Ponton-Carss A, Hutchison C, Violato C. Assessment of communication, professionalism and surgical skills in an objective structured performance-related examination (OSPRE): A psychometric study. Am J Surg 2011;202:433-40.

25 Davis $D$, Lee $G$. The use of standardized patients in the plastic surgery residency curriculum: teaching core competencies with objective structured clinical examinations. Plast Reconstr Surg 2011;128:291-8.

26 Griesser MJ, Beran MC, Flanigan DC, et al. Implementation of an objective structured clinical exam (OSCE) into orthopedic surgery residency training. I Surg Educ 2012;69:180-9.

27 Razavi D, Merckaert I, Marchal S, et al. How to optimize physicians' communication skills in cancer care: results of a randomized study assessing the usefulness of post training consolidation workshops. J Clin Oncol 2003;21:3141-9.

28 Levinson $W$, Roter $D$. The effects of two continuing medical education programs on communication skills of practicing primary care physicians. J Gen Intern Med 1993:8:318-24.

29 Baile WF, Lenzi R, Kudelka AP, et al. Improving physician-patient communication in cancer care: outcome of a workshop for oncologists. J Cancer Educ 1997:12:166-73.

30 Brown JB, Boles M, Mullooly JP, et al. Effect of clinician's communication skills training on patient satisfaction: a randomized, controled trial. Ann Intern Med 1999.131.822-9.
31 Lenzi R, Baile WF, Berek J, et al. Design, conduct and evaluation of a communication course for oncology fellows. J Cancer Educ 2005;20:143-9.

32 Didwania A, Kriss M, Cohen ER, et al. Internal medicine postgraduate training and assessment of patient handoff skills. J Graduate Med Educ 2013;394-398.

33 Downar J, Knickle K, Granton JT, et al. Using standardized family members to teach communication skills and ethical principles to critical care trainees. Crit Care Med 2012:40:1814-19.

34 Elwyn G, Edwards A, Hood K, et al. Achieving involvement: process outcomes from a cluster randomized trial of shared decision making skill development and use of risk communication aids in general practice. Fam Pract 2004:21:337-46.

35 Fallowfield $L$, Jenkins $V$, Farewell $V$, et al. Efficacy of a cancer research UK communication skills training model for oncologists: a randomised controlled trial. Lancet 2002;359:650-56.

36 Farber NJ, Friedland A, Aboff BM, et al. Using patients with cancer to educate residents about giving bad news. Palliat Care 2003;19:54-7.

37 Geeta MG, Krishnakumar P, Rajasree KC, et al. Effectiveness of communication skills training on perceptions and practice of paediatric residents. Indian J Paediatr 2011;78:979-82

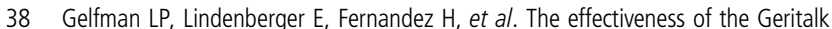
communication skills course: a real-time assessment of skill acquisition and deliberate practice. J Pain Symptom Manage 2014;48:738-744.e6.

39 Griffith $\mathrm{CH}$, Wilson JF, Langer $\mathrm{S}$, et al. House staff nonverbal communication skills and standardised patient satisfaction. J Gen Intern Med 2003:18:170-4.

40 Howells RJ, Davies HA, Silverman JD, et al. Republished paper: assessment of doctors' consultation skills in the paediatric setting: the paediatric consultation assessment tool. Postgrad Med J 2010;86:584-90.

41 Hulsman RL, Wynand JGR, Winnubst JAM, et al. The effectiveness of a computer-assisted instruction program on communication skills of medical specialists in oncology. Med Educ 2002;36:125-34.

42 Lagan $\mathrm{C}$, Wehbe-Janek H, Waldo K, et al. Evaluation of an interprofessional clinician-patient communication workshop utilizing standardized patient methodology. J Surg Educ 2012;70:95-103.

43 Parish SJ, Stein MR, Hahn SR, et al. Teaching and assessing residents' skills in managing heroin addiction with objective structured clinical exams (OSCEs). Subst Abus 2013;34:350-5.

44 Parish SJ, Ramaswamy M, Stein MR, et al. Teaching about substance abuse with objective structured clinical exams. J Gen Intern Med 2006;21:453-9.

45 Back AL, Arnold RM, Baile WF, et al. Efficacy of communication skills training for giving bad news and discussing transitions to palliative care. Arch Intern Med 2007;167:453-60.

46 Falcone JL, Claxton RN, Marshall GT. Communication skills training in surgical residency: a needs assessment and metacognition analysis of a difficult conversation objective structured clinical examination. J Surg Educ 2014;71:309-15.

47 Jefferies $A$, Simmons B, Tabak D, et al. Using an objective structured clinical examination (OSCE) to assess multiple physician competencies in postgraduate training. Med Teach 2007;29:183-91.

48 Johnson RL, Roter D, Powe NR, et al. Patient race/ethnicity and quality of patient-physician communication during medical visits. Am J Public Health 2004:94:2084-90.

49 Lienard A, Merckaert I, Libert Y, et al. Transfer of communication skills to the workplace during clinical rounds: Impact of a program for residents. PLOS ONE 2010;5:e12426.

50 Eraut M. Developing professional knowledge and competence. Philadelphia: Taylor \& Francis, 1994

51 Delvaux N. Contribution l'evaluation des effets de la formation psychologique des soignnts en oncologie [dissertation]. Brussels, Belgium: Universite Libre de Bruxelles, 1999.

52 Booth C, Maguire P. Development of a rating system to assess interaction between cancer patients and health professionals. London, UK, Report to Cancer Research Campaign; 1991:37.

53 Roter DL. The Roter Interactional Analysis System (RIAS) Coding Manual. 1990.

54 Parel M, Maguire $\mathrm{P}$, Heaven C. The development of a training model to improve health professionals' communication skills, self-efficacy and outcome expectancies when communicating with cancer patients. Soc Sci Med 1997:44:231-40.

55 The Art of Medicine ${ }^{T M}$ (cited 20 September 2014). http://www.healthcareresearch com/Art-of-Medicine.php

56 Faman JM, Paro JA, Rodriguez RM, et al. Hand-off education and evaluation: piloting the observed simulated hand-off experience (OSHE). J Gen Intern Med 2010;25:129-34

57 Horwitz LI, Dombroski J, Murphy TE, et al. Validation of a handoff assessment tool: the Handoff CEX. J Clin Nurs 2013:22:1477-86.

58 Elwyn $G$, Edwards $A$, Wensing $M$, et al. Shared decision making: developing the OPTION scale for measuring patient involvement. Qual Saf Health Care 2003; 12:93-9.

59 Ford S, Hall A, Ratcliffe D, et al. The medical interaction process system (MIPS): an instrument for analysing interviews of oncologists and patients with cancer. Soc Sci Med 2000:50:553-66.

60 Kelley AS, Back AL, Arnold RM, et al. Geritalk: communication skills training for geriatric and palliative medicine fellows. J Am Geriatr Soc 2012;60:332-7. 
61 Hulsman RL, Janssen M, Ros WJG. Handleiding scoreformulier video-opnamen. [Manual of the communication rating system]. Utrecht: Utrecht University Faculty of Medicine, 1994.

62 Wolf MH, Putnam SM, James SA, et al. The medical interview satisfaction scale: development of a scale to measure patient perceptions of physician behaviour. J Behav Med 1978;1:391-401.

63 Colliver JA, Swartz MH. Assessing clinical performance with standardized patients. JAMA 1997;278:790-1.

64 Falcone JL, Schenarts KD, Ferson PF, et al. Using elements from an acute abdominal pain Objective Structured clinical Examination (OSCE) leads to more standardized grading in the surgical clerkship for third year medical students. J Surg Educ 2011:68:408-13.
65 Hulsman RL, Ros WJG, Janssen M, et al. Interact-Cancer. The development and evaluation of a computer-assisted course on communication skills for medical specialists in oncology. Patient Educ Couns 1997;30:129-41.

66 Buyck D, Lang F. Teaching medical communication skills: a call for greater uniformity. Fam Med 2002;34:337-43.

67 Kalet A, Earp JA, Kowlowitz V. How well do faculty evaluate the interviewing skills of medical students? J Gen Intern Med 1992;7:499-505

68 Davis DA, Mazmanian PE, Fordis M, et al. Accuracy of physician self-assessment compared with observed measures of competence: a systematic review. J Am Med Assoc 2006:296:1094-102.

69 McCarney R, Warner J, Iliffe S, et al. The Hawthorne effect: a randomised, controlled trial. BMC Med Res Methodol 2007:7:30. 